\title{
Un estudio transversal sobre el modo de adquisición de la interrogativa en inglés en un grupo de estudian- tes de segundo ciclo de Educación Secundaria Obligatoria (ESO). Implicaciones educativas
}

\section{A transversal study on the way of acquisition of the interrogative in english in a group of students in the second cycle of compulsory secondary education (ESO). Educational implications}

\author{
Antonio F. LEBRÓN FUENTES \\ IES ‘Sierra de las Villas’. Villacarrillo (Jaén)
}

Recibido: Febrero 2011

Aceptado: Diciembre 2011

\section{Resumen}

Este artículo comienza con una revisión general sobre algunos de los aspectos y hallazgos más importantes en el campo de la adquisición de segundas lenguas (SLA) desde una doble perspectiva psicolingüística y didáctica. Nos referiremos a las dicotomías cognición / automatización, inducción / deducción y adquisición / aprendizaje, junto al papel de las interrogativas en la lengua hablada a través de las principales corrientes de influencia. También estudiaremos la relación entre los conceptos de construcciones transicionales e interlengua y las etapas de desarrollo de las interrogativas en conversación. Seguidamente, comentaremos la relación existente entre las interrogativas y el texto narrativo. Más tarde procederemos al análisis y descripción de la producción verbal de interrogativas por parte de un grupo de estudiantes de inglés como lengua extranjera. Nuestro estudio está basado en un corpus de 69 preguntas producidas por 63 estudiantes en el segundo ciclo de Educación Secundaria. Nuestro análisis corrobora algunas de las hipótesis actuales sobre la adquisición de las interrogativas: a) el uso de fórmulas por parte de nuestros estudiantes, y b) la coherencia de su proceso de adquisición de la interrogativa con las etapas naturales en el desarrollo de dicha estructura. Para terminar, intentaremos establecer algunas implicaciones educativas para la enseñanza de las interrogativas en Educación Secundaria.

Palabras clave: adquisición, aprendizaje, ESO, etapa de desarrollo, formula, inglés, interrogativa, pregunta Yes/No, pregunta Wh-. 


\begin{abstract}
This paper starts with a general revision on some of the most important aspects and findings in the field of Second Language Acquisition (SLA) from both a psycholinguistic and a didactic perspective. We will refer to the dichotomies cognition / automation, induction / deduction and acquisition / learning, together with the role of the interrogative in the spoken language throughout the main influential currents. We will also study the relationship between the concepts of transitional constructions and interlanguage and the stages of development of interrogatives in conversation. After that, we will comment on the relationship between the interrogatives and the narrative text. Later, we will go on with the analysis and description of the verbal production of interrogatives on the part of a group of students of English as a Foreign Language. Our study is based on a corpus of 69 questions produced by 63 students in the second cycle of Secondary Education. Our analysis corroborates some of the current hypotheses on the acquisition of interrogatives: a) the use of formulas on the part of our students, and b) the coherence of their process of acquisition of the interrogative with the natural stages in the development of this structure. To finish, we will try to set some educational implications for the teaching of interrogatives in Secondary Education.
\end{abstract}

Keywords: acquisition, learning, CSE, development stage, formula, English, interrogative, Yes/No question, Wh- question.

\title{
El papel de la interrogativa en la adquisición del inglés como segunda lengua (sla)
}

Las interrogativas son unas estructuras gramaticales esenciales en la adquisición de la lengua extranjera por parte de nuestros estudiantes de Secundaria. En la mayoría de los casos, las programaciones de inglés de Secundaria las incluyen e integran en un lugar determinado de dichas programaciones de acuerdo con el nivel de dificultad que presentan a la hora de su aprendizaje, contemplándolas como meras estructuras lineales.

Aquí nos proponemos sugerir una visión diferente del papel de las interrogativas en el estudio y adquisición del inglés como segunda lengua. Pretendemos mostrar que dicho papel, como el de cualquier otra forma gramatical, puede y debe ser estudiado también desde el punto de vista del discurso hablado (conversación). Para ello, y tras una breve reflexión sobre algunos aspectos generales en el campo de la adquisición de segundas lenguas (SLA), trataremos de exponer las etapas básicas del desarrollo de las interrogativas dentro del discurso conversacional y comentaremos, a modo de ejemplo, la relación existente entre la interrogativa y el texto narrativo, con el fin de hacer caer en la cuenta al lector del hecho de que la interrogativa también puede desarrollarse y, de hecho se desarrolla, aunque en menor medida, en otros tipos de textos diferentes del propiamente conversacional o discursivo.

Todo ello, nos servirá para tratar de indagar en las posibles implicaciones pedagógicas que pueda tener el papel jugado por la interrogativa en la adquisición del inglés en nuestro alumnado de Educación Secundaria. 


\section{Tres dicotomías esenciales}

Desde una perspectiva psicolingüística, el estudio de la adquisición de una segunda lengua (SLA) se ha caracterizado por los intentos de definir dicotomías tales como cognición / automatización, inducción / deducción, y adquisición / aprendizaje.

Las raíces del concepto automatización hay que buscarlas en la psicología conductista, y en última instancia en las teorías del psicólogo ruso Ivan Pavlov, para quien el proceso de aprendizaje consistía en la formación de asociaciones entre estímulos y respuestas. Por otro lado, cognición tiene mucho que ver con Ausubel y su teoría del aprendizaje cognitivo. Según dicha teoría el aprendizaje es una “...clearly articulated and precisely differentiated conscious experience that emerges when potentially meaningful signs, symbols concepts, or propositions are related to and incorporated within a given individual's cognitive structure on a non arbitrary and substantive basis." ("...experiencia consciente claramente articulada y diferenciada con precisión que emerge cuando los signos potencialmente significativos, los símbolos, los conceptos, o las proposiciones están relacionados con y se incorporan dentro de la estructura cognoscitiva de un individuo dado en una base substantiva y no arbitraria.") (Anderson y Ausubel, 1965, p. 8).

Ambos términos, automatización y cognición, pueden resultar de utilidad al hablar del proceso de adquisición de la segunda lengua, sobre todo, si tenemos en cuenta el hecho de que dichos términos se pueden relacionar directamente con otras dos dicotomías: adquisición / aprendizaje e inducción / deducción. De acuerdo con la trilogía aquí propuesta, podemos establecer una relación entre las tres dicotomías mencionadas hasta ahora según la figura 1:

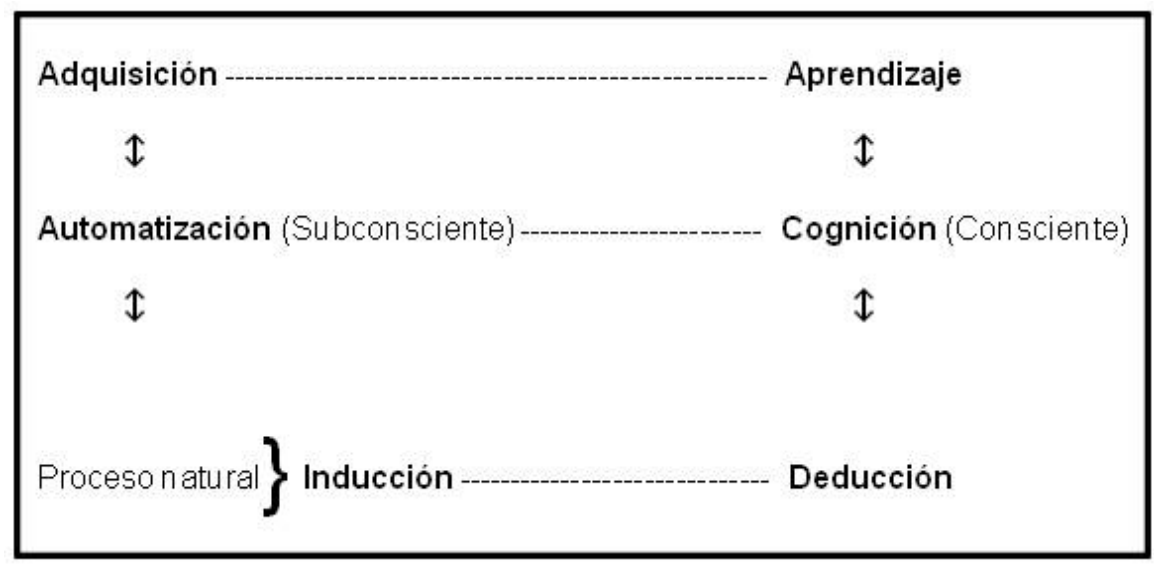

Figura 1. Relación entre las dicotomías adquisición / aprendizaje, automatización / cognición e inducción / deducción.

De aquí se desprende que la adquisición de una lengua es un proceso natural de automatización subconsciente llevado a cabo mediante procesos inductivos, mientras que 
el aprendizaje de una lengua envuelve procesos conscientes (algo que ya había apuntado Ausubel, 1965) y deductivos.

Para Krashen (1985), los aprendices adultos tienen dos maneras de aumentar su nivel de competencia en una segunda lengua: adquisición, que es subconsciente y se basa en una comunicación significativa y decidida con hablantes de la lengua objeto; y aprendizaje, que consiste en la internalización de reglas explícitas bajo control consciente. Ambos procesos son independientes y mutuamente excluyentes.

Otros teóricos, por ejemplo, McLaughlin y Bialystok (ver en Brown, 1987, p. 190 y ss.), creen que no es difícil adquirir algo que hemos aprendido por medio de un control consciente, convirtiendo la distinción anterior en algo poco claro y difícil de demostrar.

En la figura 2 hemos tratado de dar un paso más intentando ejemplificar de una manera gráfica el modo en que los procesos de inducción y deducción se relacionan con el desarrollo natural de la segunda lengua:

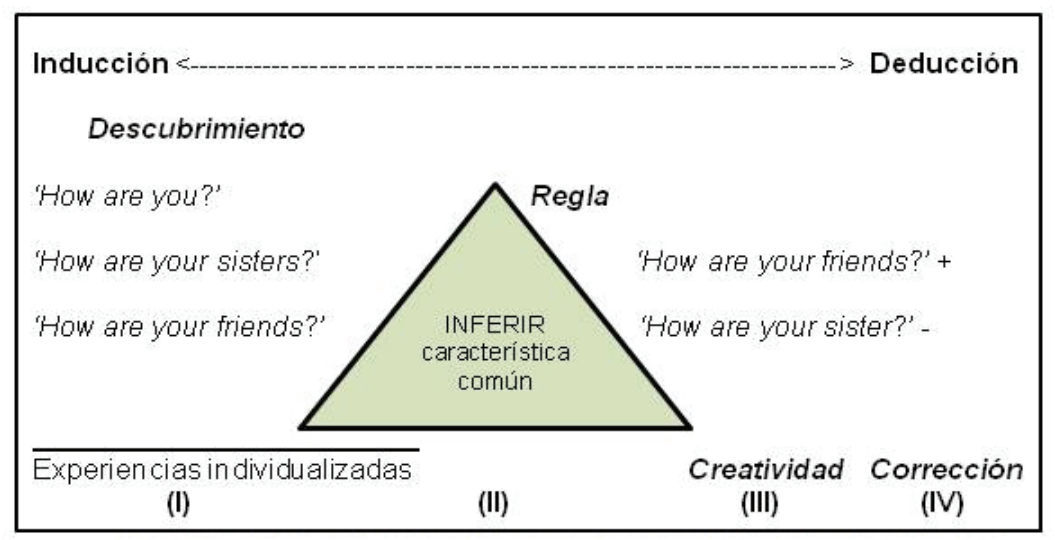

Figura 2. Inducción y deducción en relación con el desarrollo natural de la segunda lengua.

Por medio de la inducción y una vez que el individuo ha memorizado algunos ejemplos individuales (I), la persona es capaz de inferir la característica común que caracteriza dichos ejemplos individuales, extrayendo de este modo la regla general (II) antes de intentar adivinar la regla y estimulando así su creatividad lingüística (III). La etapa final será una etapa de corrección o retroalimentación, inducida por el oyente (IV).

La deducción parte de la regla, dada dicha regla, junto con sus excepciones, directamente por el profesor, suprimiendo de este modo los pasos (I) y (II), y por tanto, los automatismos. La inducción es más natural y lógica que la deducción. 
Para que exista inducción, necesitamos 'input' en forma de ejemplos. Por ello, no podemos olvidarnos de hacer referencia al concepto de input, fundamental en la hipótesis propuesta por Krashen (1985): "The Input hypothesis claims that humans acquire language in only one way - by understanding messages, or by receiving 'comprehensible input'." ("La hipótesis del input afirma que los humanos adquirimos la lengua de una única manera - comprendiendo mensajes, o recibiendo 'input comprensible'.') (p. 2).

Dicho concepto está íntimamente relacionado con la existencia de oportunidades para comunicarse e interactuar. Al mismo tiempo, dicho input debe ser comprensible y estimular de este modo el output del individuo (Krashen, 1985). El problema principal desde nuestro punto de vista viene a ser el de demostrar qué es realmente un input comprensible.

De cualquier modo, lo que sí es cierto es que ambos procesos inductivo y deductivo tienen una importancia fundamental en el desarrollo natural de la segunda lengua, siendo dicho desarrollo una conjunción de inducción y deducción. No en vano, Rivers (1983) afirma: "In a teaching situation both induction and deduction may be very effective, depending on the way they are integrated into the total teaching-learning situation ... We cannot imitate without activating a cognitive process." ("En una situación de aprendizaje ambas la inducción y la deducción pueden ser muy efectivas, dependiendo del modo en que se integren en la situación global de enseñanza-aprendizaje... No podemos imitar sin activar un proceso cognitivo.") (p. 91).

Como hemos visto a lo largo de toda esta sección, el intento de fijar barreras precisas entre los procesos de adquisición y aprendizaje plantea numerosos problemas, ya que, en multitud de ocasiones, los mecanismos que dichos procesos implican suelen superponerse; y la barrera que los separa se hace difusa y difícil de demostrar. Por ello, a partir de aquí, usaremos el término adquisición como sinónimo de aprendizaje. En el título también se ha usado dicho término según acabamos de especificar.

\section{La interrogativa: estructura básica de la lengua}

Para contemplar la interrogativa desde una perspectiva didáctica, nos referiremos a ella brevemente a través de tres de las principales teorías gramaticales surgidas durante el siglo XX. (Para un estudio más detallado de éstas y otras teorías gramaticales, consúltese Brown, 1987).

- El estructuralismo considera las interrogativas y la oración en general desde el punto de vista de la lingüística. De acuerdo con los seguidores de esta corriente, el estudio de las estructuras es fundamental para adquirir una segunda lengua. Consecuentemente, muchas gramáticas en esta etapa están basadas en el estudio de las formas, sin tener en cuenta los aspectos léxicos o pragmáticos.

- Los generativistas ven las interrogativas como estructuras superficiales resultantes de la aplicación de reglas transformacionales a una estructura profunda. Por ejemplo, si aplicamos ciertas reglas transformacionales a la estructura pro- 
funda 'I like potatoes' (simple, declarativa, activa), podemos obtener la estructura superficial interrogativa 'Do you like potatoes?'. Lo importante aquí es la habilidad para aplicar ciertas reglas transformacionales. Dicha habilidad es incluso más importante que el resultado, lo cual se ha enfatizado por dicha corriente.

- El funcionalismo añade el factor 'función' al hablar sobre la oración. Para la gramática funcional, la interrogativa no es una mera forma en sí misma, sino una forma usada para codificar una función. Por tanto, ahora debemos hablar sobre sugerencias ('Why don't we go to the cinema?'), peticiones de información ('Do you know where is the bus station?'), ofrecimientos ('Shall I clean your car?'), etc., funciones éstas llevadas a cabo mediante el uso de interrogativas $-\mathrm{y}$, por supuesto, por otros tipos de estructuras.

\section{La interrogativa en la lengua hablada}

Hasta ahora hemos considerado a las interrogativas como oraciones lineales. Pero hoy en día todo el mundo está de acuerdo con la idea de que la lengua se desarrolla dentro del discurso (Hatch, 1978). Por tanto, la interrogativa ha de desarrollarse - si hablamos en términos de lengua hablada - dentro del discurso hablado.

La figura 3 muestra el papel de la interrogativa en la conversación:

\section{Competencia comunicativa \\ - Competencia gramatical \\ --> Oración (Emisión). Proposición. \\ - Competencia discursiva \\ $\rightarrow->$ Texto HATCH---> Conversación \\ - Competencia sociolingüistica \\ - Competencia sociocultural \\ - Competencia estratégica}

Figura 3. Papel de la interrogativa en la conversación.

Como sabemos, una de las sub-categorías que integran la competencia comunicativa según Canale y Swain (1980) es la competencia gramatical. Dicha sub-competencia se traduce en el uso de una serie de oraciones o emisiones lingüísticas que incorporadas al texto discursivo han de integrarse en la conversación. De este modo, no nos resultará 
difícil insertar las interrogativas dentro del discurso, como forma de contribución a la adquisición del inglés como segunda lengua en Educación Secundaria.

Hatch (1978) propone pasar de la frase aislada al discurso. Como ejemplo, podemos decir que la idea expresada en el ejemplo 'Draw three red apples in the basket' puede ser inducida de la siguiente conversación:
Pregunta: 'Do you like apples?'
Respuesta: 'Yes'.
Pregunta: 'What colour?'
Respuesta: 'Red'.
Pregunta: 'How many?'
Respuesta: 'Three'.
Pregunta: 'Where?'
Respuesta: 'In the basket'.

Como vemos en nuestro ejemplo, la explotación de la interrogativa dentro de un discurso es extensiva y contextualizada. La novedad cuando queremos secuenciar una programación en Educación Secundaria consiste en tener en cuenta los criterios discursivos. El diálogo será abierto, con libertad para introducir elementos diferentes, estimulando de este modo la creatividad individual del alumnado.

\section{Construcciones transicionales e interlengua}

Una vez llegados a este punto, podemos introducir la relación entre los términos construcciones transicionales e interlengua, y el desarrollo de las interrogativas en relación con dichos conceptos.

Las construcciones transicionales, como las llaman Dulay, Burt y Krashen (1982), "are the language forms learners use while they are still learning the grammar of a language" - ("son las formas que los aprendices usan mientras se encuentran en el proceso de aprendizaje de la gramática de la lengua") (p.121). De acuerdo con esto, construcciones transicionales del tipo interrogativo 'Why you no come?' o 'What those are?' son indicadores del progreso que han conseguido los estudiantes en su intento de descifrar y producir un nuevo sistema lingüístico. Son, en sí mismas, un reflejo del sistema de interlengua del aprendiz.

Brown, en el capítulo 9 de su obra Principles of Language Learning and Teaching (Brown, $1987^{2}$, pp.168-186), expone las etapas del desarrollo de la interlengua, las fuentes de error y las estrategias de comunicación que usa el estudiante a la hora de adquirir una lengua.

Etapas de desarrollo de las interrogativas

Dulay, Burt y Krashen ya habían sistematizado las etapas en el proceso de adquisición de las Wh-questions (Dulay, Burt y Krashen, 1982, pp. 127-130). Pero a nosotros nos parece más interesante la sistematización llevada a cabo por Littlewood (1984), para quien las interrogativas, tanto las yes/no questions, como las Wh- questions, sufren tres etapas de desarrollo sin transiciones repentinas en el desarrollo real del aprendiz. 
Dichas etapas, que pasamos a representar de una manera gráfica a continuación, se superponen considerablemente:

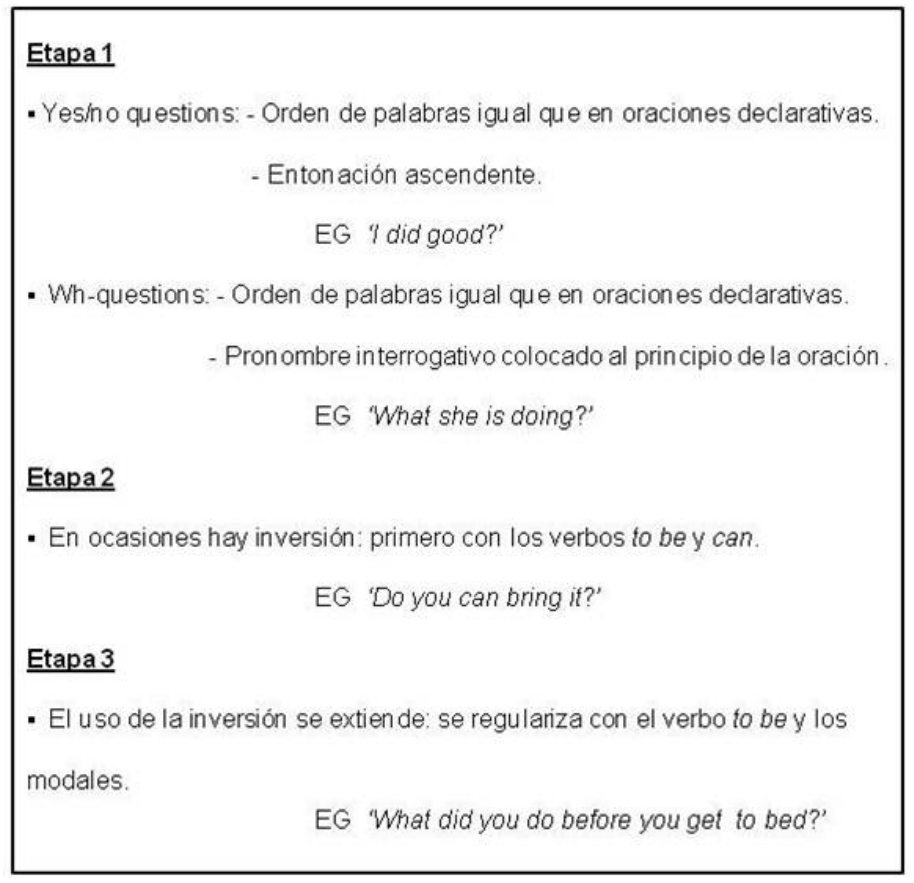

Figura 4. Etapas de desarrollo de las interrogativas. Adaptado de Littlewood (1984: 44-45).

\section{Interrogativas y el texto narrativo}

Para terminar nuestra aproximación al estudio del papel de la interrogativa en la adquisición del inglés como segunda lengua, realizaremos un breve comentario sobre la relación entre ésta y el texto narrativo.

Biber (1989) define los tipos de textos sobre la base de criterios estrictamente lingüísticos (similitudes en el uso de características lingüísticas que se dan en el texto). Distingue en su análisis entre exposición narrativa general y narrativa imaginativa. Por otra parte, los géneros se definen y se distinguen sobre la base de criterios metalingüísticos sistemáticos. Se cree que la narración es el género más universal porque todas las culturas tienen tradiciones narrativas. La narración supone una muy buena oportunidad para que nuestros estudiantes de Secundaria usen las interrogativas y desarrollen su competencia comunicativa.

Para concluir esta sección, veamos un ejemplo de la transcripción de una secuencia narrativa producida por un alumno de la profesora Luque Agulló (1997), que ha estudiado el discurso oral narrativo en el aprendizaje del idioma Inglés y sus implica- 
ciones didácticas en la enseñanza primaria: “- Take the one sip and y tenía- son (con cálculos mentales)- fifty nine- take another sip and y tenia (risas)- thirty-nine- take another sip and tenía (risas) nineteen // the man she 'go to the cinema with me?' she'oh yes- but-but go to my house and change my dress' // the man is unhappy // ya no sé más-"(Luque Agulló, 1997, p. 371).

La interrogativa que contiene esta secuencia narrativa tiene su contexto apropiado dentro de la narración. Por ello, la utilidad del discurso narrativo para la enseñanza de las interrogativas y, al mismo tiempo, para la adquisición de la competencia comunicativa puede verificarse claramente.

\section{Estudio transversal de las producciones interrogativas en inglés del grupo de es- tudiantes seleccionado}

La adquisición de la interrogativa ha sido objeto de investigación en numerosos estudios (por ejemplo, Felix, 1981; Dulay, Burt y Krashen, 1982; Littlewood, 1984; Ellis, 1984). Sin embargo, quizá dos de los estudios que arrojan más luz sobre la adquisición de las interrogativas del inglés como lengua extranjera en el contexto formal de la enseñanza sean: el de Felix (1981), de cuyo estudio de aprendices de inglés en un contexto escolar alemán se desprende que hay algunos estudiantes que son capaces de usar formas idiosincráticas de interrogativas que no se les había enseñado antes; y el de Torras i Cherta (1993), llevado a cabo con 72 alumnos de $8^{\circ}$ de la antigua Educación General Básica (EGB), donde viene a demostrar, entre otras cuestiones, que sólo cuando el alumnado ha explotado las 'rutinas o fórmulas lingüísticas' hasta la saciedad es cuando éste empieza a producir lenguaje creativo que refleja las etapas naturales en la adquisición de la interrogativa.

Risueño Martínez (2003) estudió los efectos de la intervención explícita en la enseñanza de las estructuras interrogativas inglesas en estudiantes universitarios de la titulación de Maestro en la especialidad de Lengua Extranjera (Inglés), concluyendo que dicha intervención tuvo efectos positivos tanto en la corrección gramatical, como desde el punto de vista de la secuencia de adquisición y que los efectos positivos derivados del programa de instrucción no son temporales, sino que son duraderos.

Moya Guijarro, también en 2003, realizó un estudio por edades con alumnos de Educación Infantil y Primaria (3-8 años) sobre la adquisición/aprendizaje de la pronunciación, del vocabulario y de las estructuras interrogativas en lengua inglesa, advirtiendo la semejanza que existe entre el proceso de adquisición de las estructuras interrogativas en español y en inglés.

El trabajo de Rowland (2007) trata de explicar los errores cometidos por los aprendices a la hora de adquirir la interrogativa, mientras que el de Tornyova y Valian (2009) se centra en el proceso de inversión del auxiliar en las preguntas Wh-. 
Creemos que, a pesar del interés de los estudios citados, es necesario explorar el modo de adquisición de las estructuras interrogativas del inglés por parte de nuestros estudiantes.

Por consiguiente, el propósito de este estudio es el análisis y la descripción de la producción verbal de interrogativas por parte de un grupo de estudiantes de Inglés como lengua extranjera en el segundo ciclo de Educación Secundaria Obligatoria (ESO), ya que dicha investigación nos permitirá, por un lado, confirmar nuevamente algunas de las hipótesis que ya se barajaban sobre esta cuestión; y, por otro lado, adecuar nuestros métodos de enseñanza de las estructuras interrogativas a nuestro contexto particular educativo, dentro del nuevo currículo de la Educación Secundaria Obligatoria (ESO).

\section{Metodología}

\section{Población}

Los sujetos de este estudio son 63 estudiantes españoles de inglés del segundo ciclo de Educación Secundaria Obligatoria (ESO). La selección de los mismos se llevó a cabo de una manera aleatoria dentro de un grupo de adolescentes de 14 y 15 años de edad, sin hacer distinción de sexo, teniendo en cuenta tan sólo el hecho de estar escolarizados en el segundo ciclo de la ESO, y por tanto, haber recibido cierta instrucción formal sobre la formación y usos de la interrogativa dentro del contexto escolar. El investigador, profesor no habitual de los educandos, tuvo que solicitar el consentimiento de cada uno de los participantes seleccionados para este estudio.

\section{Procedimiento y métodos}

Previamente a nuestro estudio, obtuvimos un corpus de transcripciones pertenecientes al grupo de estudiantes mencionado más arriba. A dichos aprendices se les había pedido que realizaran una entrevista en inglés al recopilador. Debían imaginar que dicha entrevista era una actividad para la revista de su centro educativo (en caso de que dicha revista existiese) o para algún proyecto para la clase de inglés. También valía cualquier otra idea que se les ocurriese.

Con el fin de que no se quedasen en blanco a la hora de preguntar, se les entregó un guión que contenía las instrucciones aquí descritas y un cuadro con 10 pistas en español a modo de sugerencia, con el objetivo de ayudarles a formular al menos una pregunta para cada una de las pistas. El guión incluía las siguientes pistas, tal como se transcriben:
1. Nombre
2. ¿Qué tal?
3. Apellidos
4. Ocupación, trabajo,...
5. ¿Tienes ... 

6. ¿Te gustó...
7. La edad o de dónde es
8. Dato de algún familiar o amigo,...
9. Verano pasado
10. Pregunta libre

De este amplio corpus de transcripciones de emisiones interrogativas producidas por dicho grupo de estudiantes, se seleccionaron las producciones correspondientes a la pista 10. Pregunta libre.

Tipo de análisis.

De acuerdo con la naturaleza de los datos, hemos realizado un análisis cuantitativo o estadístico y otro cualitativo o interpretativo. El procedimiento usado para este segundo tipo de análisis ha consistido en interpretar las emisiones de los participantes tomando como base los hallazgos más importantes en el campo de la adquisición de segundas lenguas, sobre todo, aquéllos que tratan de explicar las etapas naturales de adquisición de las interrogativas en inglés, con el fin de observar el modo en que los estudiantes adquieren estas oraciones.

\section{Resultados}

Datos del corpus

A continuación transcribimos el contenido íntegro del corpus utilizado en nuestro estudio.

\begin{tabular}{|c|c|c|c|c|}
\hline $\begin{array}{l}\mathrm{N}^{0} \text { de } \\
\text { estu- } \\
\text { diante }\end{array}$ & Emisión & Tipo & Estructura & Tema \\
\hline 1 & Do you like study / (Er) Spanish? & FA & $\mathrm{Y} / \mathrm{N}$ & Gustos \\
\hline $\begin{array}{l}2 \mathrm{a} \\
\mathrm{b} \\
\mathrm{c}\end{array}$ & $\begin{array}{l}\text { Do you like music? } \\
\text { What music? } \\
\text { Do you like "Take that"? I love }\end{array}$ & $\begin{array}{l}\text { FA } \\
\text { E } \\
\text { FA }\end{array}$ & $\begin{array}{l}\mathrm{Y} / \mathrm{N} \\
\mathrm{Wh}- \\
\mathrm{Y} / \mathrm{N}\end{array}$ & $\begin{array}{l}\text { Gustos } \\
\text { Gustos } \\
\text { Gustos }\end{array}$ \\
\hline $\begin{array}{c}3 a \\
b\end{array}$ & $\begin{array}{l}\text { Er / do you like Spain? } \\
\text { And Jaen? }\end{array}$ & $\begin{array}{c}\text { FA } \\
\text { E }\end{array}$ & $\begin{array}{l}\mathrm{Y} / \mathrm{N} \\
\mathrm{Y} / \mathrm{N}\end{array}$ & $\begin{array}{l}\text { Gustos } \\
\text { Gustos }\end{array}$ \\
\hline $\begin{array}{c}4 a \\
b\end{array}$ & $\begin{array}{l}\text { Do you have any hobby? } \\
\text { Do you have a friend? }\end{array}$ & $\begin{array}{l}\text { FA } \\
\text { FA }\end{array}$ & $\begin{array}{l}\mathrm{Y} / \mathrm{N} \\
\mathrm{Y} / \mathrm{N}\end{array}$ & $\begin{array}{l}\text { Tener } \\
\text { Tener }\end{array}$ \\
\hline 5 & Where you start? & FA & $\mathrm{Y} / \mathrm{N}$ & --- \\
\hline 6 & What type of music do you like? & FA & Wh- & Gustos \\
\hline $\begin{array}{l}7 \mathrm{a} \\
\mathrm{b}\end{array}$ & $\begin{array}{l}\text { Where / where / where are your / friends from? } \\
\text { Where, where are you / where do you live in } \\
\text { Jaen, here? }\end{array}$ & $\begin{array}{l}\text { FA } \\
\text { FA }\end{array}$ & $\begin{array}{l}\text { Wh- } \\
\text { Wh- }\end{array}$ & $\begin{array}{l}\text { Origen } \\
\text { Origen }\end{array}$ \\
\hline 8 & Did you like it? & FA & $\mathrm{Y} / \mathrm{N}$ & Gustos \\
\hline
\end{tabular}




\begin{tabular}{|c|c|c|c|c|}
\hline 9 & Do you like Jaen? & FA & $\mathrm{Y} / \mathrm{N}$ & Gustos \\
\hline 10 & $\begin{array}{l}\text { What I do now? What /// I do now? } \\
\text { Si, yes. What are you doing? }\end{array}$ & FA & Wh- & Trabajo \\
\hline 11 & Do you like Spanish food? & FA & $\mathrm{Y} / \mathrm{N}$ & Gustos \\
\hline 12 & Have you / any girlfriend? & FA & $\mathrm{Y} / \mathrm{N}$ & Tener \\
\hline 13 & Have you got a boyfriend? & FA & $\mathrm{Y} / \mathrm{N}$ & Tener \\
\hline 14 & How old are you? & $\mathrm{R}$ & Wh- & Edad \\
\hline 15 & Do you like a job? Tu trabajo & FA & $\mathrm{Y} / \mathrm{N}$ & Gustos \\
\hline $\begin{array}{c}16 a \\
b\end{array}$ & $\begin{array}{l}\text { Have your hair colour / natural colour? } \\
\text { Are you sure? }\end{array}$ & $\begin{array}{c}\text { FA } \\
\text { R }\end{array}$ & $\begin{array}{l}\mathrm{Y} / \mathrm{N} \\
\mathrm{Y} / \mathrm{N}\end{array}$ & $\begin{array}{l}\text { Tener } \\
\text { Tener }\end{array}$ \\
\hline 17 & How long have you here? & FA & Wh- & --- \\
\hline 18 & Have you any brothers / or sisters? & FA & $\mathrm{Y} / \mathrm{N}$ & Tener \\
\hline 19 & Have you any pets? & FA & $\mathrm{Y} / \mathrm{N}$ & Tener \\
\hline 20 & Where are you from? & $\mathrm{R}$ & Wh- & Origen \\
\hline 21 & Where do you live? & $\mathrm{R}$ & Wh- & Lugar res. \\
\hline 22 & What is your favourite colour? & $\mathrm{R}$ & Wh- & Gustos \\
\hline 23 & Do you like coffee? & FA & $\mathrm{Y} / \mathrm{N}$ & Gustos \\
\hline 24 & What is your address? & $\mathrm{R}$ & Wh- & Dirección \\
\hline 25 & $\begin{array}{l}\text { What is your phone number? /// number phone } \\
\text { (o como sea eso)? /// (ruidos) /// What is your } \\
\text { telephone number? }\end{array}$ & $\mathrm{R}$ & Wh- & Teléfono \\
\hline 26 & Would you like to have a drink? & FA & $\mathrm{Y} / \mathrm{N}$ & Gustos \\
\hline 27 & Do you like the music /// the disco? & FA & $\mathrm{Y} / \mathrm{N}$ & Gustos \\
\hline 28 & $\begin{array}{l}\text { What's your favourite team of the / Profes- } \\
\text { sional Champions League of football of Spain? }\end{array}$ & FA & Wh- & Gustos \\
\hline 29 & Like you no, do you like football everydays? & FA & $\mathrm{Y} / \mathrm{N}$ & Gustos \\
\hline 30 & Did like the cinema? & FA & $\mathrm{Y} / \mathrm{N}$ & Gustos \\
\hline 31 & Is it raining? & FA & $\mathrm{Y} / \mathrm{N}$ & Ref. ext. \\
\hline 32 & Where is Tom? & FA & $\mathrm{Y} / \mathrm{N}$ & Ref. ext. \\
\hline 33 & Can you play the piano? & FA & $\mathrm{Y} / \mathrm{N}$ & Habilidad \\
\hline 34 & Is Maria dancing in his house? & FA & $\mathrm{Y} / \mathrm{N}$ & Ref. ext. \\
\hline 35 & Do you like / go surfing? & FA & $\mathrm{Y} / \mathrm{N}$ & Gustos \\
\hline 36 & $\begin{array}{l}\text { And do you like Spain and Spanish people, } \\
\text { typical? Because Spanish people and Austra- } \\
\text { lian people are very different? }\end{array}$ & FA & $\mathrm{Y} / \mathrm{N}$ & Gustos \\
\hline 37 & $\begin{array}{l}\text { What / what kind / kind / the music do you like } \\
\text { it? }\end{array}$ & FA & Wh- & Gustos \\
\hline 38 & Would you like / your / profession? & FA & $\mathrm{Y} / \mathrm{N}$ & Gustos \\
\hline 39 & What / is / your / surname? & $\mathrm{R}$ & Wh- & Nombre \\
\hline 40 & Where are you from? & $\mathrm{R}$ & Wh- & Origen \\
\hline 41 & Did I like / did I like the cinema? & FA & $\mathrm{Y} / \mathrm{N}$ & Gustos \\
\hline
\end{tabular}




\begin{tabular}{|l|l|l|l|l|}
\hline 42 & // What // are you from? & R & Wh- & Origen \\
\hline 43 & How old are you? & R & Wh- & Edad \\
\hline 44 & $\begin{array}{l}\text { Eh // how // how old / are you? How old are } \\
\text { you? }\end{array}$ & R & Wh- & Edad \\
\hline 45 & Do you like dance? & FA & Y/N & Gustos \\
\hline 46 & How old are you? & R & Wh- & Edad \\
\hline 47 & Have you got a summer /// a sister? & FA & Y/N & Tener \\
\hline 48 & What's the time? & R & Wh- & Ref. ext. \\
\hline 49 & Do you play football? & FA & Y/N & Habilidad \\
\hline 50 & Do you think for me? & FA & Y/N & -- \\
\hline 51 & Have you got a girl friend? & FA & Y/N & Tener \\
\hline 52 & Have you got a girlfriend? & FA & Y/N & Tener \\
\hline 53 & Have you got any cat? & FA & Y/N & Tener \\
\hline 54 & How old /// how old are you? & R & Wh- & Edad \\
\hline 55 & Do you like the cinema? & FA & Y/N & Gustos \\
\hline 56 & Are you married? & R & Y/N & Estado c. \\
\hline 57 & Do you like go to the discotheque? & FA & Y/N & Gustos \\
\hline 58 & What are your hobbies? & FA & Wh- & Gustos \\
\hline 59 & Er /// did you like your job? & FA & Y/N & Gustos \\
\hline 60 & Do you like potatoes? & FA & Y/N & Gustos \\
\hline 61 & Er /// er /// do you like this school? & FA & Y/N & Gustos \\
\hline 62 & /// do you like playing tennis? & FA & Y/N & Gustos \\
\hline 63 & How much it cost? (señalando a un bolígrafo) & FA & Wh- & Ref. ext. \\
\hline
\end{tabular}

Los datos están organizados en cinco columnas del modo siguiente:

- Número de estudiante: contiene el número de orden que se ha asignado de forma aleatoria a cada participante.

- Emisión: contiene la transcripción literal de la producción lingüística correspondiente a la pista 10. Pregunta libre de cada uno de los participantes.

- Para la transcripción hemos adoptado las siguientes convenciones:

/ Pausa respiratoria corta (vacía)

// Pausa respiratoria media (vacía)

// Pausa respiratoria larga (vacía)

Er Pausa llena

() Anotaciones sobre el contexto o significado realizadas por el investigador 
- Tipo: Expresa el tipo o clase de emisión de acuerdo con las siguientes abreviaturas:

- FA: Fórmula abierta (sección de lenguaje sin analizar más una secuencia de lenguaje creativo y libre).

- E: Pregunta elíptica (ejemplos idiosincráticos caracterizados por la omisión de elementos que se consideran necesarios para la construcción de una determinada estructura).

- R: Rutina (también fórmula cerrada; lenguaje rutinario que se aprende como un todo).

- Estructura: Contiene la clasificación de las emisiones en preguntas Yes/No (Y/N; se espera respuesta afirmativa o negativa) y preguntas $\mathrm{Wh}-(\mathrm{Wh}-$; se espera recibir la información sobre lo que se pregunta en la respuesta).

- Tema: Contiene la adscripción de las emisiones a un determinado tema de conversación, siendo Lugar res. (Lugar de residencia), ref. ext. (referencia externa); y --- (sin determinar).

Si se desea una explicación más completa de las definiciones de elipsis, preguntas Yes/No y preguntas Wh-, consúltese la obra de Quirk, Greenbaum, Leech y Svartvik. (1985). Para una justificación de la clasificación del tipo de emisiones en fórmulas abiertas o rutinas, véase Tejada Molina (1993).

\section{Análisis de los resultados}

Para el presente estudio se ha analizado una selección de 69 preguntas, emitidas por 63 escolares. El gráfico 1 hace referencia al porcentaje de preguntas elípticas, rutinas y fórmulas abiertas empleadas por los estudiantes. Las preguntas elípticas son producciones idiosincráticos caracterizadas por la omisión de elementos que se consideran necesarios para la construcción de una determinada estructura. En nuestro estudio sólo aparecen dos preguntas elípticas: 'What music?' ( $\left.\mathrm{n}^{\mathrm{o}} 2 \mathrm{~b}\right)$ y 'And Jaen?' ( $\left.\mathrm{n}^{\mathrm{o}} 3 \mathrm{~b}\right)$. Las rutinas son lenguaje rutinario que se aprende como un todo. Dentro de este concepto podemos clasificar emisiones como, por ejemplo, 'What is your address?' $\left(\mathrm{n}^{\circ}\right.$ 24) o 'How old are you?' ( $\mathrm{n}^{\mathrm{o}} 26$ ). Las fórmulas abiertas constan de una sección de lenguaje sin analizar más una secuencia de lenguaje creativo y libre. Ejemplos de ello son: 'What's your favourite team of the / Professional Champions League of football of Spain?' ( $\left.\mathrm{n}^{\circ} 28\right)$ o '/// do you like playing tennis?' $\left(\mathrm{n}^{\circ} 62\right)$. Como podemos observar, el porcentaje de fórmulas abiertas (73'91\%) es bastante superior al de rutinas $\left(23{ }^{\prime} 19 \%\right)$ y al de preguntas elípticas $(2,9 \%)$. 


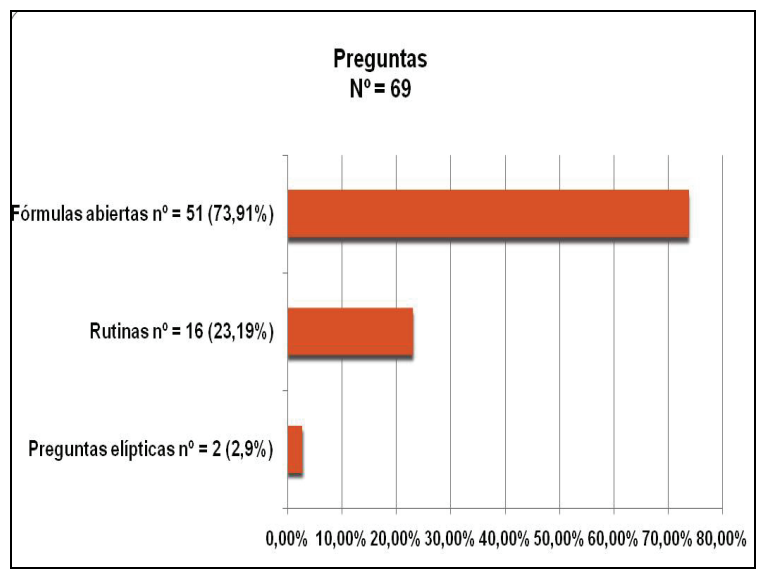

Gráfico 1. Porcentajes de las emisiones (preguntas elípticas, rutinas y fórmulas abiertas) producidas por los estudiantes.

El gráfico 2 representa el porcentaje de preguntas Yes/No (se espera respuesta afirmativa o negativa) y preguntas Wh- (se espera recibir la información sobre lo que se pregunta en la respuesta) emitidas por los escolares. Las preguntas Yes/No $\left(62^{\prime} 31 \%\right)$ predominan considerablemente sobre las preguntas Wh- (37'69\%). Su puntuación es casi el doble que la de las preguntas Wh-.

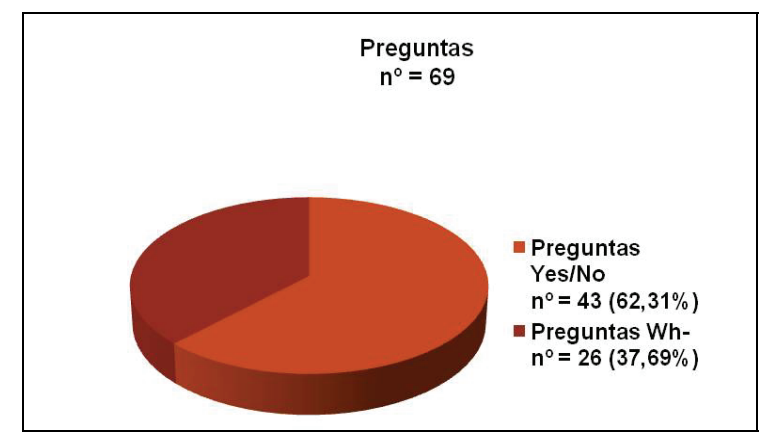

Gráfico 2. Porcentajes de preguntas Yes/No y preguntas Wh-.

El gráfico 3 reproduce los porcentajes de producciones lingüísticas por temas de conversación. Junto a la especificación de cada tema, aparece el número de preguntas relacionadas con dicho tema y el porcentaje sobre el total de preguntas estudiadas $\mathrm{La}$ mayoría de las preguntas están relacionadas con el tema gustos $(44,92 \%)$ y con tener $(17,39 \%)$. El resto de temas presenta una distribución más uniforme, oscilando entre el $7,25 \%$ y el $1,45 \%$. 


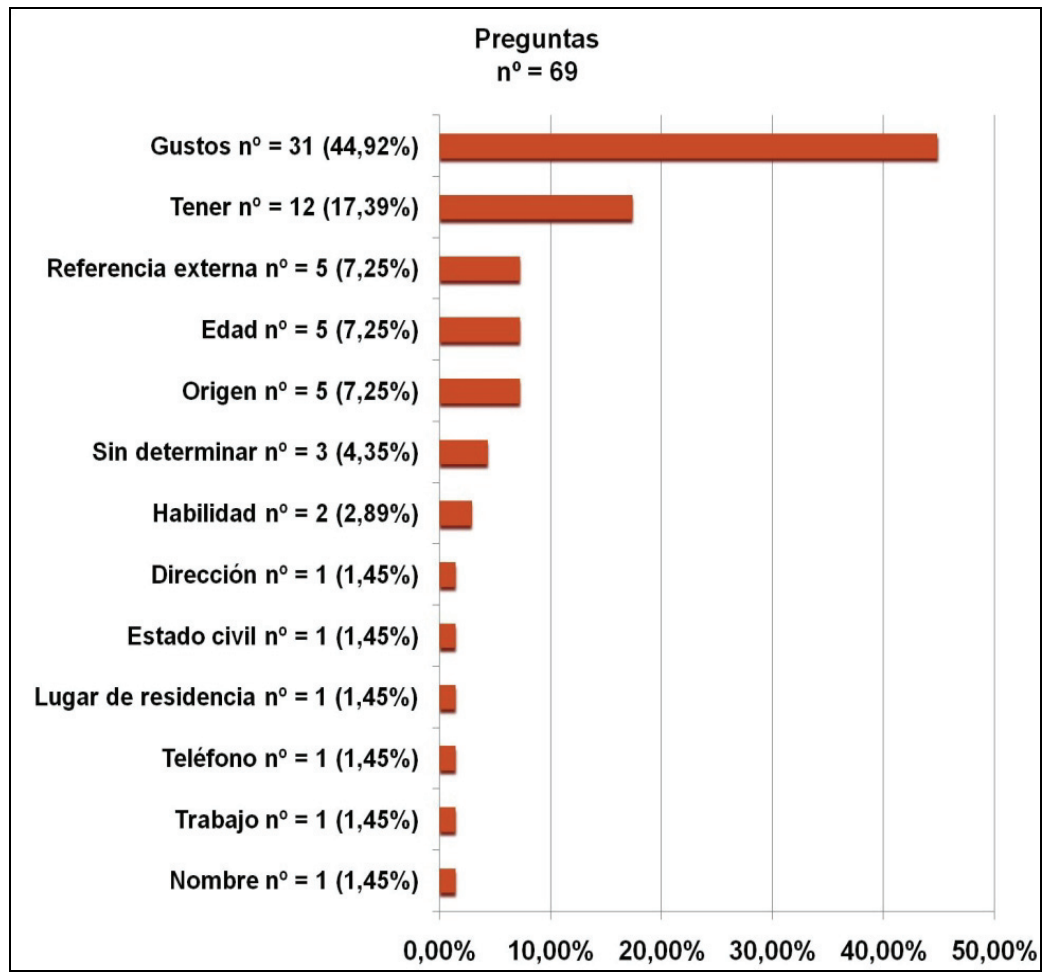

Gráfico 3. Porcentajes en la adscripción de las emisiones a los temas de conversación.

El gráfico 4 corresponde a la distribución de las emisiones de acuerdo con las etapas de desarrollo de las interrogativas. Para establecer dichas etapas hemos seguido a Littlewood. Littlewood (1984, pp. 44-45) distingue cuatro etapas en el desarrollo de las preguntas Yes/No y Wh-:

\section{-Etapa 1:}

- Preguntas Yes/No: - Orden de palabras igual que en oraciones declarativas.

- Entonación ascendente.

Ejemplo: 'I did good?'

- Preguntas Wh- : - Orden de palabras igual que en oraciones declarativas.

- Pronombre interrogativo colocado al principio de la oración.

Ejemplo: 'What she is doing?'

-Etapa 2: - En ocasiones hay inversión: primero con los verbos to be y can.

Ejemplo: 'Do you can bring it?'

-Etapa 3: - El uso de la inversión se extiende: se regulariza con el verbo to be y los modales.

Ejemplo: 'What did you do before you get to bed?' 
Nuestro alumnado se encuentra, salvo dos excepciones ('Where you start?'- $\mathrm{n}^{\mathrm{o}} 5 \mathrm{y}$ 'How much it cost?' $-\mathrm{n}^{\mathrm{o}}$ 63), en la etapa 3 de desarrollo de la interrogativa $(97,1 \%)$.

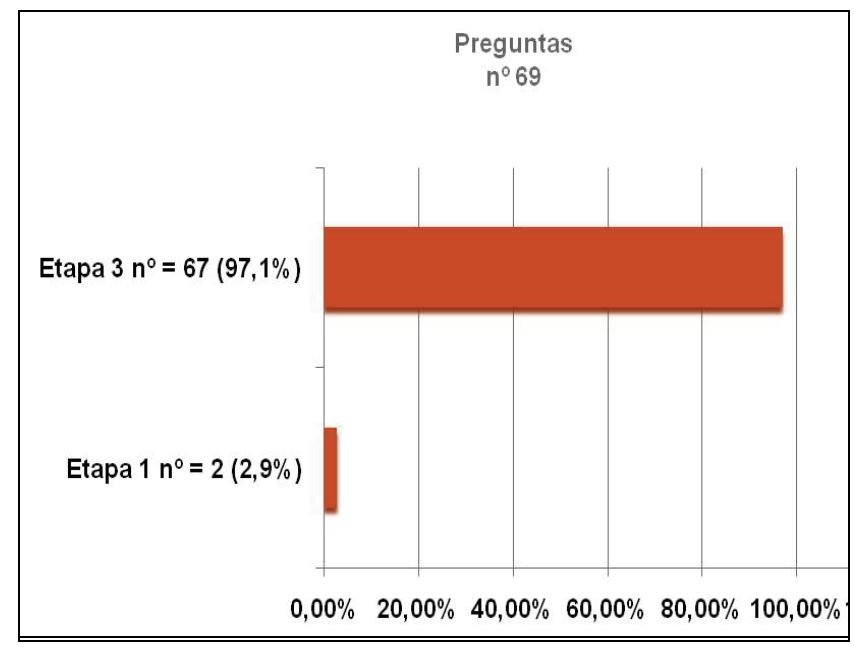

Gráfico 4. Distribución de las emisiones de acuerdo con las etapas en la adquisición de las interrogativas.

En relación con la operación sintáctica de la inversión, hemos de señalar aquí que Santelmann, Berk, Austin, Someshakar, Lambert y Lust (2002) demuestran que la competencia gramatical para realizar dicha operación está presente desde las primeras producciones en sujetos infantiles.

El gráfico 5 recoge el porcentaje de preguntas bien o mal formuladas desde una perspectiva gramatical. Como podemos apreciar en el mismo, el porcentaje de preguntas gramaticalmente correctas $\left(73^{\prime} 91 \%\right)$ casi duplica el número de aquéllas cuya formulación presenta algún tipo de error estructural (26’09\%).

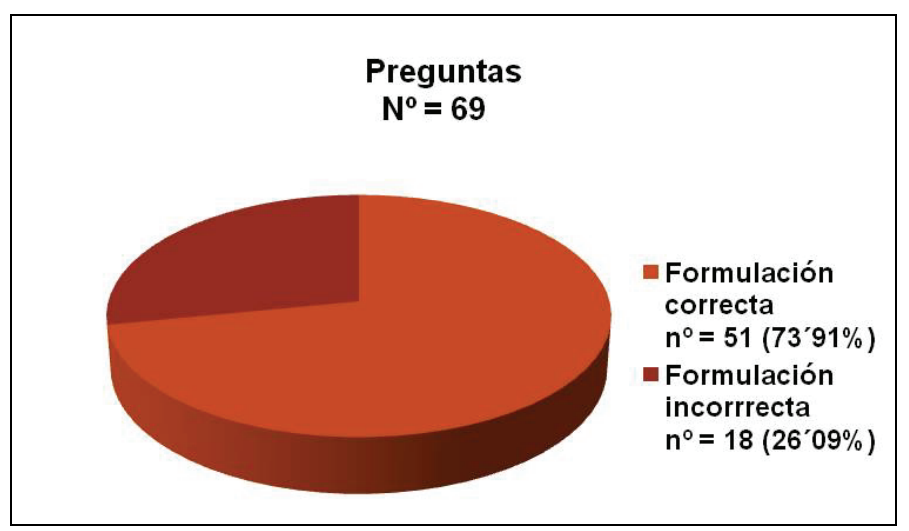

Gráfico 5. Porcentaje de corrección gramatical en las emisiones de los estudiantes. 


\section{Discusión}

Según el gráfico 1, podemos observar que el porcentaje de fórmulas abiertas $\left(75^{\prime} 36 \%\right)$ es bastante superior al de rutinas $\left(21^{\prime} 74 \%\right)$ y al de preguntas elípticas. Esto se debe al hecho de que sólo unos cuantos estudiantes son incapaces de usar una fórmula abierta cuando se les pide que formulen una pregunta de tema libre. Por otra parte, el alto porcentaje de fórmulas abiertas muestra cómo la mayor parte de nuestro alumnado ha adquirido la destreza necesaria para expandir sus emisiones creativamente, tomando como punto de partida, en la mayoría de los casos, los patrones típicos a los que han sido expuestos en los centros de Educación Secundaria. Las emisiones 'What music?' and 'And Jaen?' están clasificadas como preguntas elípticas ("ejemplos idiosincráticos caracterizados por la omisión de elementos que se consideran necesarios para la construcción de una determinada estructura." ); y no pueden considerarse dentro de ninguno de los otros dos grupos estrictamente hablando.

En relación con la estructura básica utilizada: Yes/No o Wh- (ver gráfico 2), hemos de decir que la disparidad de puntuación entre las preguntas Yes/No (que representan el $62,31 \%$ del total) y las preguntas Wh- (representadas sólo por el $37^{\prime} 69 \%$ ) viene dada principalmente por el uso extendido de las preguntas Yes/No 'Do you like...?' y 'Have you got...?', cuya adquisición por parte del alumnado resulta más fácil que en el caso de las preguntas Wh-.

De la observación del gráfico 3, deducimos que la mayoría de los temas están muy relacionados con las pistas sugeridas a nuestro alumnado. Este resultado viene marcado por el hecho de que, a pesar de que nuestro objeto de estudio es la pista 10. Pregunta libre, nuestro alumnado está muy condicionado a usar el resto de las pistas y, de hecho, así lo hace. La puntuación es considerablemente más alta en el tema 'gustos' $(44,92 \%)$, donde se observa una gran predilección de los estudiantes por la utilización del verbo like, ya sea de una manera explícita o implícita (en las preguntas elípticas). En nuestro corpus podemos encontrar fácilmente preguntas como 'Do you like music?', 'What music?' (estudiante $\mathrm{n}^{\circ} .2$ ), Do you like Jaén?' (estudiante $\mathrm{n}^{\circ} .9$ ), Do you like a job? (estudiante $\mathrm{n}^{\circ}$. 15) o, donde los estudiantes emplearon el patrón 'Do you like + sección de lenguaje abierta?' A dicho verbo le sigue en frecuencia de uso el verbo have (que aparece en el $17^{\prime} 39 \%$ de las emisiones). Ambos verbos también están muy presentes en las programaciones educativas de los cursos de ESO, por lo que se supone que los aprendices están bastante familiarizados con ellos en el segundo ciclo de estas enseñanzas. Las secuencias de lenguaje más llamativas corresponden a las emisiones clasificadas como Referencia externa.

Lo más interesante es observar cómo, en algunos casos, el estudiante es capaz de preguntar sobre otros aspectos no especificados en las pistas que se les han sugerido. Esto demuestra la habilidad que presenta parte de nuestro alumnado de recordar patrones y temas y de ser más creativo que el resto comunicativamente hablando.

En relación con la etapa de desarrollo de las interrogativas en que nuestro alumnado se halla inmerso (ver gráfico 4), podemos decir lo siguiente: Aparentemente, casi todos 
nuestros estudiantes se encuentran en la etapa 3 (Littlewood, 1984, pp. 44-45), porque la mayor parte de ellos produjeron emisiones que presentaban inversión regular de have, do y be. Pero, realmente, creemos que el resultado, a pesar de haber sido observado dentro de un grupo de preguntas de tema libre, está muy condicionado al input previo recibido en forma de fórmulas. Por esto, un alto porcentaje de sus producciones son fórmulas abiertas, es decir, una sección de lenguaje sin analizar más una secuencia libre, en algunos casos con una pausa entre ambas secciones al intentar crear una fórmula abierta:

$$
\begin{aligned}
& \mathrm{n}^{\mathrm{o}} \text { 35.- '// Do you like / go surfing?' } \\
& \mathrm{n}^{\mathrm{o}} \text { 38.- '// Would you like / your / profession?' }
\end{aligned}
$$

Este argumento prueba el valor didáctico del uso del lenguaje de fórmulas en el aula (Tejada Molina, 1993).

El contexto de aprendizaje de nuestros estudiantes puede, por tanto, haber influenciado sus procesos de aprendizaje. Como consecuencia, el empleo de fórmulas, puede haberse visto alentado por el medio ambiente lingüístico del aula al serles requerida al alumnado una producción temprana. Dichas fórmulas, al mismo tiempo, ayudan al estudiante a sostener y extender su uso creativo de la lengua.

Sólo podemos encontrar dos preguntas Wh- que encajen claramente en la etapa 1 de desarrollo de las interrogativas, con orden de palabras igual que en las declarativas, entonación ascendente y la partícula interrogativa colocada al principio de la oración:

$\mathrm{n}^{\circ}$ 5.- 'Where you start?'

$\mathrm{n}^{\mathrm{o}}$ 63.- 'How much it cost?' (señalando a un bolígrafo)

Pensamos que este tipo de secuencias es predictible si aceptamos, como Tornyova y Valian (2009), que los aprendices producen este tipo de estructuras sin inversión en las preguntas Wh- debido a que la existencia de preguntas Yes/No sin inversión los lleva a pensar que dicha transformación pueda ser opcional también en las preguntas Wh-.

Rowland (2007) ha demostrado que los errores de inversión son más frecuentes en las preguntas Wh- que en las preguntas Yes/No.

Del análisis de la clasificación de las emisiones de los aprendices según el porcentaje de corrección gramatical en las mismas (gráfico 5) se desprende que el 73'91\% de los alumnos han adquirido ya la estructura de la interrogativa y pueden usarla de una manera correcta. El resto del alumnado $\left(26^{\prime} 09 \%\right)$ presenta ciertos problemas en su utilización, aunque se trata de dificultades mínimas y fácilmente superables en periodos de aprendizaje posteriores. 


\section{Conclusiones e implicaciones educativas}

La formulación y dominio de las estructuras interrogativas de la lengua inglesa entraña para nuestros estudiantes innumerables dificultades. Por tanto, resulta crucial por nuestra parte, como profesores de inglés, intentar comprender el modo en que nuestros aprendices adquieren este aspecto tan importante de la gramática, de tal modo que seamos capaces de proponerles modelos de enseñanza/aprendizaje que les faciliten la adquisición de dichas estructuras de un modo lógico, ordenado y coherente.

La comprensión de las etapas en la adquisición de las interrogativas en inglés puede ayudarnos en gran medida a la hora de entender las producciones de nuestros estudiantes de Educación Secundaria. Dicha comprensión nos será especialmente útil para orientar nuestra enseñanza del inglés en relación con este punto gramatical de una manera más coherente con el desarrollo natural dentro del proceso de adquisición de las interrogativas.

Nuestra visión de la interrogativa como profesores de inglés ha de ampliarse para que seamos capaces de integrar su estudio dentro del discurso, sobre todo con los estudiantes más avanzados, ya que, como se ha demostrado (Hatch, 1978; Luque Agulló, 1997), dicha estructura gramatical es susceptible, al igual que el resto de estructuras gramaticales, de ser estudiada dentro del discurso. Por ello, sería bueno idear nuestras programaciones teniendo en cuenta criterios discursivos a la hora de secuenciar los programas y proponer a nuestros estudiantes tareas dirigidas al uso de estas estructuras contextualizadas dentro del discurso, no sólo narrativo, como se deduce del fragmento transcrito del trabajo de la profesora Luque Agulló (1997), sino también dentro del diálogo, donde quizá la interrogación cobra pleno sentido dentro del discurso.

Hemos de ser conscientes de que la efectividad del aprendizaje de las interrogativas dependerá en gran medida del hecho de que el profesor sepa cómo hacer que el estudiante se percate de su capacidad para desarrollar una serie de estrategias que le permitan adquirir dichas estructuras de una manera más fácil. Esto ayudará a establecer en la clase procedimientos capaces de promover un aprendizaje más fructífero y la adquisición de las interrogativas de una manera más efectiva.

El profesor debe planificar el proceso de aprendizaje de tal modo que el estudiante reciba el máximo tiempo de exposición posible a la lengua objeto. Durante las primeras etapas de adquisición de una nueva lengua, los estudiantes muestran una tendencia a simplificar los recursos que emplean para expresarse. Una de sus estrategias es el uso de lenguaje de fórmulas (Tejada Molina, 1993), cuyo uso en nuestro corpus es bastante frecuente. Por ello, existe la necesidad de incluir este tipo de lenguaje cuando estructuremos una gramática de la lengua hablada. Estas unidades permiten a los estudiantes entrar en comunicación.

También hemos visto como el proceso de adquisición de la interrogativa por parte de los aprendices de Inglés como segunda lengua no dista demasiado del proceso natural de adquisición de dicha estructura según Littlewood (1984), ya que no resulta difícil 
encajar las emisiones de los estudiantes de Secundaria dentro de alguna de las diferentes fases de adquisición de la interrogativa propuestas por dicho lingüista.

Nuestro análisis corrobora, por tanto, dos de las hipótesis actuales sobre la adquisición de las interrogativas: a) el uso de fórmulas por parte de nuestros estudiantes, y b) la coherencia de su proceso de adquisición de la interrogativa con las etapas naturales en el desarrollo de dicha estructura.

Hasta el momento, la investigación en el área de la adquisición de la interrogativa en inglés como segunda lengua tiene más preguntas que respuestas respecto a qué es lo que pedagógicamente puede hacerse para favorecer la adquisición de dicha estructura. De los muchos interrogantes que podría dejar este estudio nos interesa mencionar uno como futuro tema de investigación: ¿Qué metodología de enseñanza/aprendizaje podría favorecer la adquisición de las interrogativas?

\section{Referencias Bibliográficas}

ANDERSON, R. C. Y AUSUBEL, D. P. (Eds.) (1965). Readings in the Psychology of Cognition. New York: Holt, Rinehart y Winston, Inc.

BIBER, D. (1989). ‘A Typology of English Texts', en Linguistics, 27, pp. 3-43.

BROWN, H. D. (1987). Principles of Language Learning and Teaching. (2a ed.) New Jersey: Prentice H. R.

CANALE, M. Y M. SWAIN (1980). 'Theoretical Bases of Communicative Approaches in Second Language Teaching and Testing', en Applied Linguistics, 1 (I), pp. 1-47.

DULAY, H., M. BURT AND S. KRASHEN (1982). Language Two. New York: OUP.

ELLIS, R. (1984). Classroom Second Language Development. Oxford: Pergamon.

FELIX, S. (1981). 'The effect of formal instruction in second language acquisition', en Language Learning 31(1), pp. 87-112.

HATCH, E. (1978). 'Discourse analysis and Second Language Acquisition'. En Hatch, E. (ed.) (1978).

HATCH, E. (ed.) (1978). Second Language Acquisition: A Book of Readings. Rowley, Mass.: Newbury House.

KRASHEN, S. D. (1985). The Input Hypothesis: Issues and Implications. London: Longman.

LITTLEWOOD, W. (1984). Foreign and Second Language Learning: Language Acquisition Research and its Implications for the Classroom. CUP. 
LUQUE AGULLÓ, G. (1997). El discurso oral narrativo en el aprendizaje del idioma Inglés. Implicaciones didácticas en la enseñanza primaria. Tesis doctoral. Universidad de Jaén.

MOYA GUIJARRO, A. J. (2003). 'La adquisición/aprendizaje de la pronunciación, del vocabulario y de las estructuras interrogativas en lengua inglesa. Un estudio por edades', en Didáctica (Lengua y Literatura) 15, pp. 161-177.

QUIRK R, S. GREENBAUM, G. LEECH Y J. SVARTVIK. (1985). A Comprehensive Grammar of the English Language. London: Longman.

RISUEÑO MARTÍNEZ, J.J. (2003). La adquisición de la interrogativa en inglés, estudio longitudinal sobre la incidencia de una intervención explícita. Tesis doctoral. Universidad de Jaén.

RIVERS, W. M. (1983). Communicating Naturally in a Second Language: Theory and Practice in Language Teaching. New York: CUP.

ROWLAND, C. F. (2007). 'Explaining errors in children's questions', en Cognition, 104, pp. 106-134.

SANTELMANN L., BERK S., AUSTIN J., SOMESHAKAR S., LAMBERT, K. Y LUST B. (2002). 'Continuity and development in the acquisition of inversion in yes/no questions', en Journal of Child language 29 (4), pp. 813-840.

TEJADA MOLINA, G. (1993). 'Las primeras unidades en la enseñanza de las lenguas extranjeras', en Greta, 1(1), pp.11-18.

TORRAS I CHERTA, M. R. (1993). 'Un estudio transversal del nivel de adquisición de la interrogación en inglés con alumnos de $8^{\circ}$ de E.G.B.', en Revista Española de Lingüística Aplicada, 9, pp. 169-187.

TORNYOVA, L., \& VALIAN, V. (2009). 'The Role of Cross-Linguistic Variation in the Acquisition of Auxiliary Inversion in Wh- questions', en Proceedings of GALANA 2008, pp. 282-290.

Correspondencia con el autor

Antonio F. Lebrón Fuentes

IES 'Sierra de las Villas'. Villacarrillo (Jaén)

Plaza Requena, 9

Baeza 23440, Jaén

e-mail : antoniofe15@yahoo.es 